\title{
Ableitung der chemischen Verwandtschaft aus der Elektronentheorie.
}

\author{
Von Heinrice Teddt. \\ Mit 18 Figuren in Text.
}

1. Unterschied zwischen einachsigen und strahlenförmigen Molekülen.

In meiner vor kurzem erschienenen Arbeit „Die Ableitung des periodischen Systems der chemischen Elemente aus der Elektronentheorie" "1) habe ich abgeleitet, daß die Perioden des natürlichen Systems der chemischen Elemente dadurch gebildet werden, daB sämtliche Elemente einer Horizontallreihe ein inneres Elektronensystem mit einer gleich großen Anzahl Elektronen haben, während sämtliche Elemente einer Vertikalreihe eine gleich große Anzahl Valenzelektronen haben, die auBerhalb des inneren Elektronensystems stehen.")

Nach den dort gemachten Ausführungen besteht das innere Elektronensystem bei den Elementen der ersten Horizontalreihe aus eineu Elektronenring, bei den Elementen der zweiten Horizontalreihe aus zwei Elektronenringen, bei den Elementen der dritten Horizontalreihe aus drei Elektronenringen, bei den Elementen der vierten Horizontalreihe aus vier Elektronenringen usw. Diese inneren Elektronenringe stellen

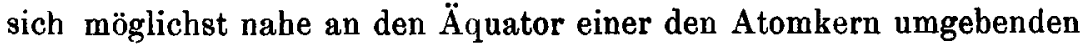
Kugelfläche ein, so daß die beiden Pole dieser Kugelfäche frei von Klektronen bleiben. Die von dem Atomkern ausgehenden positiven Kraftlinien können daher an den beiden Polen aus dem Inneren des Atoms heraus zu den Valenzelektronen gelangen (vgl. die Figuren $1 \mathrm{a}$ und $1 \mathrm{~b}$ ). Je größer die von den Elektronen freie Stelle an den Polen ist, desto mehr Kraftlinien können durch diese Stelle hindurch zu den Valenzelektronen treten. Da nun zwei Atome dadurch zu einem Molekül vereinigt werden, daß sie mit Hülfe dieser Kraftlinien mit ein und denselben Valenzelektronen verbunden werden, so wird diese Verbindung um so fester, je mehr Kraftlinien durch die von Elektronen freien Stellen an den Polen der Atome zu den

1) Z. anorg. u. allg. Ch. 106 (1919), 189-208.

2) a. a. O. S. 114 . 
Valenzelektronen gehen können. Wie ein Vergleich der Figur la mit $1 \mathrm{~b}$ ohne weiteres erkennen läBt, wird aber diese elektronenfreie Stelle an jedem der Pole um so kleiner, je mehr Elektroneuringe das innere Elektronensystem hat. Daher wird die Menge der rom Atomkern zu den Valenzelektronen gelangenden Kraftlinien ron

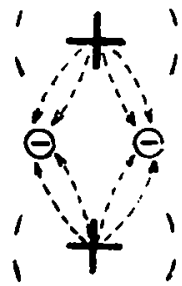

Fig. 1 a.

Molekül eines Elementes der zweiten Horizontalreibe.

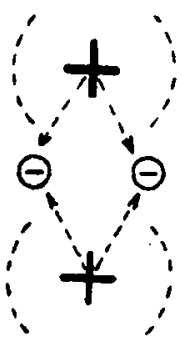

Fig. 1 b.

Molekül eines Eleunentes der sechsten Horizontalreihe

Horizontalreihe zu Horizontalreihe kleiner, und in Übereinstimm ung hiermit nimmt auch die Energie, mit der die chemischen Elemente Verbindungen eingehen, von Horizontalreihe zu Horizontalreihe in der Regel ab.

Zu den Aqusnahmen von dieser Regel gehören insbesondere die Alkalimetalle und die Metalle der alkalischen Erden. Bei diesen

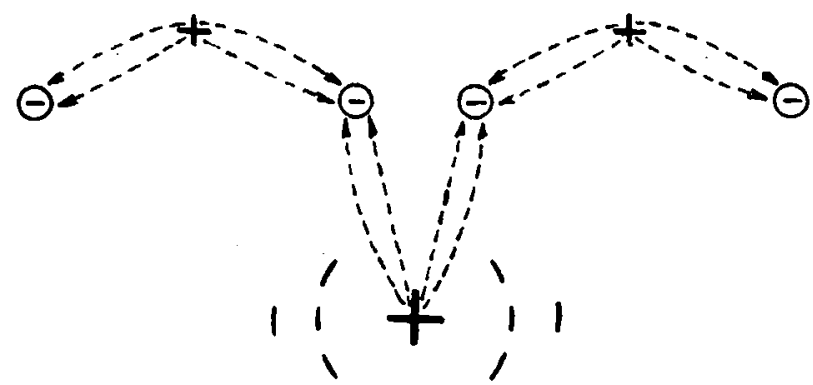

Fig. 2.

Teil eines $\mathrm{AsH}_{3}$ Moleküls (das dritte H-Atom ist auberhalb. der Papierebene im Raume liegend zu denken).

können die Elektronen, wie im Kapitel 4 abgeleitet werden wird, so leicht zurückgedrängt werden, da $B$ die eben gemachten Ausführungeu für diese Metalle keine Gültigkeit haben.

Ein Beispiel für die Verbindung eines Atoms mit zwei anderen Atomen liefert die Fig. 14, die das erst weiter unten zu besprecheude

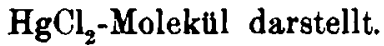


Wenn sich mit einem Atom mehr als zwei andere Atome verbinden, so müssen sich dem einen der elektronenfreien Pole dieses Atoms zwei oder mehr andere Atome mit ihren Valenzelektronenringen gegenüberstellen (vgl. den in Fig. 2 skizzierten Teil eines $\mathrm{AsH}_{3} \cdot$ Moleküls). Bei diesem Molekül können sich die drei H-Atome nicht alle drei in die verlängerte Mittellinie des As-Atoms einstellen, sondern müssen sich um dieselbe herum gruppieren. Wie Fig. 2 zeigt, werden daher die Valenzelektronen nur an dem Teile ihrer Kreisbahn von positiven Kraftlinien getroffen, der sich oberhalb des Poles des A8-Atoms befindet.

Nach dem Vorhergehenden muB man zwischen zwei verschiedenen Arten von Molekülen unterscheiden, nämlich zwischen einachsigen Molekülen, bei denen die Kerne der miteinander verbundenen Atome in einer Achse liegen (Fig. 1a, 1 b und 14), und strahlenförmigen Molekülen, bei denen die Verbindungslinien der Atomkerne strahlenförmig von einem mittleren Atom ausgehen, wie vom AsAtom in Fig. 2. Die von den Atomkerneu ausgehenden und durch die Pole hindurchgehenden positiven Kraftlinien treffen bei den einachsigen Molektulen den ganzen Umfang der Valenzelektronenringe, bei den strahlenförmigen Molekülen dagegen nur einen Teil der Valenzelektronenringe. Daher sind die Atome bei einem strahlenförmigen Bau des Molekuls weniger fest miteinander verbunden als bei einachsigen Molekülen. Da nun drei oder mehrwertige Atome strahlenförmige Moleküle bilden, zeigen diese nur geringe Bildungswärmen, vgl. $\mathrm{NH}_{3}, \mathrm{PH}_{3}, \mathrm{AsH}_{3}, \mathrm{SbH}_{3}$. Auch haben die strahlenförmig gebsuten Moleküle

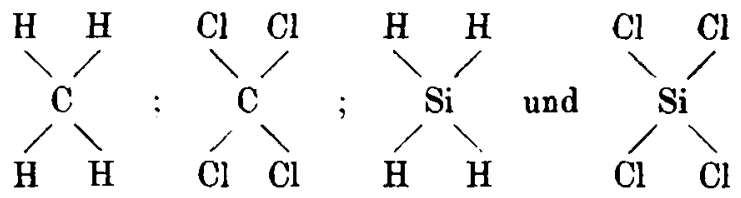

erheblich geringere Bildungswärmen als die einachsigen Moleküle O-C-O und O-Si-O. Auch die Chlorstickstoffverbindungen bilden Beispiele für die eben abgeleitete Regel:

Die Wirkungen der in diesem Kapitel abgeleiteten Regelu werden aber in vielen Fällen dadurch abgeändert, daB die Atome in einem Molekül nicht nur durch Valenzelektronen, sondern auch noch durch andere Kräfte zusammengehalten werden, die im Folgenden abgeleitet werden sollen. 


\section{Unterschied zwischen Metallatomverbindung und Metalloidatomverbindang.}

Die eben erwähnten Kräfte, welche außer den Valenzelektronen noch zur festeren Aneinanderkupplung zweier im Molekül vereinigter Atome dienen, entstehen dadurch, daB in den zum inneren Elektronensystem gehörenden Elektronenringen die Stellen, welche zwischen deu einzelnen Elektronen liegen, durch die vom Atomkern ausgehenden Kraftlinien elektrisch positiv werden. Die einander gegenïberstehenden Elektronenringe zweier in einem Molekül verbundener A tome werden sich daher so gegeneinander einzustellen suchen, daB
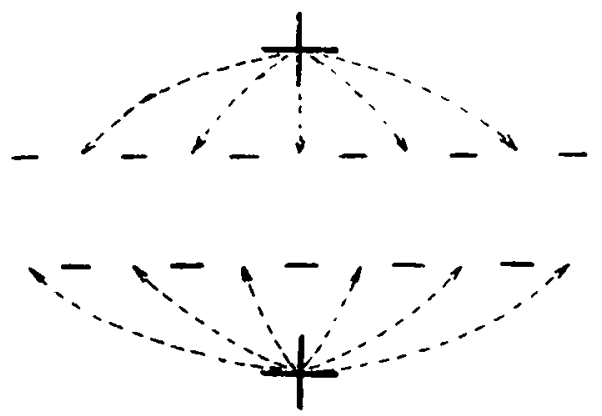

Fig. 3.

Linander gegenüberstebende Elektronenringe zweier Atome in die Papierebene aufgerollt gedacht.

immer die positiven Stellen des zu dem einen Atom gehörenden Elektronenringes den Elektronen des zu dem anderen Atom gehörenden Elektronenringes gegenüberstehen, vgl. Fig. $3 .{ }^{1}$ ) Infolgedessen werden die beiden Atome nicht nur mit Hilfe der Valenzelektronen zusammengehalten, sondern auch durch die gegenseitigen Anziehungskräfte, welche die positiven Stellen und negativen Elektronen der einander gegenüberstehenden Elektronenringe der beiden Atome aufeinander ausüben.

Den eben abgeleiteten Anziehungskräften wirien aber noch $\mathrm{Ab}$ stobungskräfte entgegen, die dadurch zustande kommen, dab die Elektronensysteme der beiden Atome und die zwischen ihnen kreisenden Valenzelektronen sich gegenseitig abstoBen. Diese AbstoBungskräfte

3) Der Ubersichtlichkeit wegen sind die Valenzelektronen in den Figuren 3 bis 9 nicht mit gezeichnet. 
werden um so stärker, je mehr die Atome sich einander näheru und verhindern, dab die beiden Atome durch die eben abgeleiteten Anziehungskräfte ganz ineinander gezogen werden.

Die von den Valenzelektronen anf die Elektronen der beiden miteinander verbundenen Atome ausgeübten AbstoBungskräfte sind um so größer, je größer die Zahl der Valenzelektronen ist. Diese Zahl ist nun bei mehrwertigen Verbindungen gröBer als bei einwertigen. Deshalb zerfallen die Moleküle organischer Verbindungen besonders leicht an denjenigen Stellen, an denen Kohlenstoffatome in mehrfacher Bindung aneinander stehen.

Die Anziehungskräfte, welche durch die bei Fig. 3 abgeleitete Gruppierung der Elektronen entstehen, kommen bei den Metalloiden kräftig, bei den Metallen aber nur schwach oder gar nicht zur Wirkung. Denn bei den Metallen sitzen die Elektronen weniger fest am Atomkern und werden daher zurückgedrängt durch die AbstoBungskräfte, welche das Elektronensystem eines gegenüber befindlichen Atoms und die Valenzelektronen auf sie ausüben (vgl. Fig. 4). Je mehr aber die beiden einander gegenüberstehenden Elektronenringe der beiden Atome auf diese Weise auseinander gedrängt werden, desto weniger kommt die gegenseitige Anziehung ihrer positiven und negativen Stellen zur Geltung.

Auberdem bewirken die von den Atomkernen ausgehenden positiven Kraftlinien, daB der Raum zwischen den Atomen an den Stellen, an denen sich nicht gerade Valenzelektronen befinden, elektrisch positiv wird; und je mehr er elektrisch positiv wird,

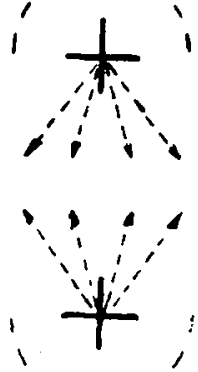

Fig. 4.

Aus zwei Metall. atomen bestehendes Molekül. desto mehr vernichtet er die Anziehungskrälte, die von den negativen Stellen des einen Atoms auf die positiven Stellen des gegenüberliegenden Atoms ausgeübt werden.

In den Molekülen der Metalle werden daher die Atome infolge des eben abgeleiteten positiven $Z$ wischenraumes weniger stark zusammengehalten als in den Metalloidmolekülen. Aus diesem Grunde treten die Metalle in Dampfform und in verdünnten Lösungen häufig einatomig auf; während die Moleküle der Metalloide meist zwei oder mehrere Atome aufweisen, weil die Anziehungskräfte der Valenzelektronen bei den Metalloiden noch vermehrt werden durch die positiven Stellen und negativen Elektronen der aneinander gegenüberstehenden Elektronenringe. 


\section{Verbindungen der Letalloide antereinander.}

Sämtliche Metalloide stehen in der rechten Hälfte des periodischen Systems. Bei diesen rechts stehenden Elementen kommt zu dem inneren Elektronenring eines jeden Atoms noch ein äuBerer Elektronenring bei denjenigen Verbindungen hinzu, bei denen nicht alle Valenzelektronen von dem Atomkern fort in eine Valenzstellung gehen. $\left.{ }^{1}\right)$ So hat $z$. B. ein Kohlenstoffatom vier Valenzelektronen, von denen im $\mathrm{CO}_{2}$-Molekül alle vier, im CO-Molekül dagegen nur zwei in eine Valenzstellung gehen, während die anderen beiden am Atomkern zurückbleiben und den eben genannten äuBeren Elektronenring bilden.

Da die zum inneren Elektronensystem gehörenden Elektronen im Atom sich zwischen dem eben besprochenen äuBeren Elektronenring und dem Atomkern befinden, können nur wenige der von dem letzteren ausgehenden positiven Kraftlinien zu den äuBeren Elektronenringen gelangen. Daher überwiegen in den äuBeren Elektronenringen die negativen Ladungen, und wenn zwei mit äuberen Elektronenringen versehene Atome in einem Molekul nebeneinander stehen, so suchen die negativen äuBeren Elektronenringe sich gegenseitig abzustoben und vermehren die im vorhergehenden Kapitel angegebenen AbstoBungskräfte.

Vergleichen wir $\mathrm{z}$. B. die eben genannten $\mathrm{CO}_{3}$ - und CO-Moleküle. Das O-A tom hat entsprechend seiner Stellung im periodischen System 6 Valenzelektronen, von denen bei jeder der eben genannten Verbindungen $z w e i$ in die Valenzstellung gehen, so daB an jedem O-A tomkern ein äuBerer Elektronenring von je vier Elektr onen zurückbleibt. Das C-Atom hat nun, wie oben gezeigt, keinen auberen Elektronenring im $\mathrm{CO}_{2}$-Molekül, dagegen ist im CO-Molekul ein äußerer Elektronenring vorhanden. Daher wird der äußere Elektronenring eines O-Atomes und mit ihnen das Atom selbst nur im CO-Molekül, nicht aber im $\mathrm{CO}_{2}$-Molekül durch einen äuBeren Elektronenring des C.Atoms von diesem abgestoben. Demnach hat das $\mathrm{CO}_{2}$-Molekul deshalb eine höhere Verbindungswärme als das $\mathrm{CO}$ Molekül, weil die Anziehungskräfte, mit denen die Atome in Molekül zusammengehalten werden, nur im CO-Molekül, nicht aber im $\mathrm{CO}_{2}$ Molekül durch einen am C-Atom vorbandenen äußeren Elektronenring geschwächt werden. Ebenso hat $\mathrm{N}^{2} \mathrm{O}^{5}$ eine böhere Bildungsenergie als $\mathrm{N}_{2} \mathrm{O}_{3}, \mathrm{~A}_{2} \mathrm{O}_{5}$ eine höhere Bildungsenergie als $\mathrm{As}_{2} \mathrm{O}_{3}, \mathrm{SO}_{3}$

1) a. a. O. \$. $193-200$ u. 207 . 
als $\mathrm{SO}^{2}$, weil nur bei den niederen, nicht aber bei den höheren Sauerstoffverbindungen an dem N- oder As- oder S-Atom noch ein äuBerer Elektronenring vorhanden ist, der die äußeren Elektronenringe der O-Atome abstöBt.

Durch die vorher im Kapitel 1 angegebene Zunahme der Zahl der inneren Elektronenringe von Horizontalreihe zu Horizontalreihe wird zwischen den Elementen der ersten Horizontalreihe einerseits und den Elementen der folgenden Horizontalreiben andererseits ein

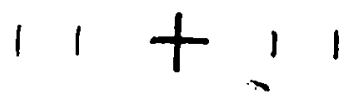

Fig. 5.

Metalloidatom der ersten Horizontalreibe mit suBerem Elektronenring.

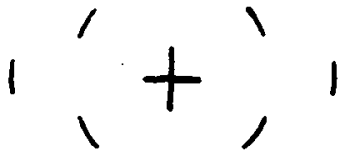

Fig. 6 .

Metalloidatom der zweiten Horizontalreihe mit äuBerem Elektronenring.

Gegensatz geschaffen, weil bei der ersten Horizontalreihe sämtliche zum Atom gehörende Teile (nämlich der Atomkern, der eine Elektronenring des inneren Systems und der etwa noch hinzukommende äuBere Elektronenring) in einer Ebene liegen (Fig. 5), während in den folgenden Horizontalreihen zwei oder mehr Elektronenringe zu beiden Seiten der Ebene liegen, die durch den Atomkern und deh äuBeren Elektronenring gebildet wird (Fig. 6).

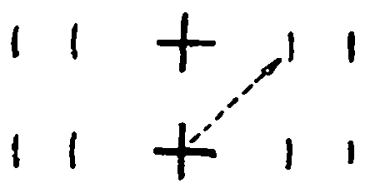

Fig. 7.

Aus zwei Metalloidstomen der ersten Horizontalreibe bestehendes Molekül.

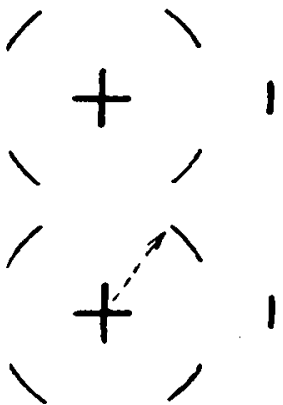

Fig. 8.

Aus 2wei Metalloidatomen der zweiten Horizontalreihe bestehendes Malekül.

Diese zu beiden Seiten der Mittelebene des Atoms vorhandenen Elektronenringe bewirken nun, daB die beiden mittleren Atomebenen zweier miteinander verbundener Atome bei den Elementen der zweiten und folgenden Horizontalreihen weiter voneinander entfernt sind, 
als bei den Elementen der ersten Horizontalreihe. Ein Vergleich der Figg. 7 und 8 läBt diesen Unterschied deutlich erkennen.

Da nun die äußeren Elektronenringe zweier miteinander verbundener Atome vorwiegend AbstoBungskräfte aufeinander ausüben, müssen diese Abstoßungskräfte in der ersten Horizontalreihe größer sein als in den folgenden, weil, wie die Figg. 7 und 8 zeigen, die äußeren Elektronenringe zweier verschiedener Atome bei den Elementen der ersten Horizontalreihe näher aneinander liegen als bei den Elementen der folgenden Horizontalreihen. Infolge dieser größeren AbstoBungskräfte können die Moleküle der in der ersten Reihe stehenden Metalloide leichter auseinindergerissen werden als die Moleküle der Elemente in den folgenden Reihen. Je leichter aber die Atome aus den Molekülen herausgerissen werden können, desto reaktionsfähiger ist das betrefiende Element. Die Elemente der ersten Horizontalreihe müssen demnach reaktionsfähiger als die der folgenden Reihen sein.

Von den Elementen der ersten Horizontalreihe bleiben in äuBeren Elektronenringe zurück: bei den dreiwertigen Stickstoffverbindungen zwei Elektronen, bei den zweiwertigen Sauerstoffverbindungen vier und bei den einwertigen Fluorverbindungen sechs Elektronen. In dieser Weise nimmt innerhalb jeder Horizontalreihe die Zah1 der Elektronen in den äußeren Elektronenringen der rechtsstehenden Elemente von links nach rechts zu. Mit zunehmender Elektronenzahl nehmen auch die AbstoBungskräfte, die diese äuBeren Elektronenringe aufeinander ausüben, von links nach rechts zu; und damit wächst auch die Leichtigkeit, mit der die Atome aus den Molekülen gerissen werden können. Somit muß auch die chemische Reaktionsfähigkeit der Metalloide innerhalb jeder Horizontalreihe von links nach rechts zunehmen. Daher ist Chlor reaktionsfähiger ais Schwefel, Fluor reaktionsfähiger als Sauerstoff und Sauerstoff reaktionsfähiger als Kohlenstoff. Wenn man auch noch Stickstoff und Phosphor zum Vergleich heranziehen will, so muB man in Rechnung ziehen, daB die meisten Verbindungen dieser Elemente strahlenförmige Moleküle haben, in denen, wie hier im Kapitel 1 gezeigt, andere Verhältnisse herrschen als in den einachsigen Molekülen.

Da nun nach den eben gemachten Ausführungen die Reaktionsfähigkeit der Elemente in der ersten Horizontalreibe am größten sein und außerdem innerhalb jeder Horizontalreihe von links nach rechts zunehmen muB, so müssen von allen Elementen diejenigen 
am reaktionsfähigsten sein, welche am rechten Ende der ersten Horizontalreibe stehen; es sind dies Sauerstoff und Fluor. Während nun diese beiden Elemente mit fast allen anderen Elementen beständige Verbindungen eingehen, sind sie nicht fähig, sich untereinander zu verbinden, weil die gegenseitige Abstoßung ihrer äußeren Elektronenringe infolge der relativ groBen Zahl der in ihnen enthaltenen Elektronen größer wird als die Anziehungskräfte, die $\mathrm{zwischen}$ einem Sauerstoffatom und einem Fluoratom vorhanden sind. Zwar ist beim Schwefel und Chlor die Zahl der in den äuBeren Elektronenringen vorhandenen Elektronen ebenso groB wie beim Sauerstoff und Fluor. Aber bei der zweiten Horizontalreihe sind die äuBeren Elektronenringe zweier miteinander verbundenen Atome, wie der Vergleich zwischen Figg. 7 und 8 zeigt, weiter entfernt voneinander, so daB die AbstoBungskräfte, die sie aufeinander ausüben, weniger zur Wirkung kommen. Deshalb können sich Schwefel und Chlor im Gegensatz zu Sauerstoff und Fluor miteinander verbinden.

Ein weiterer EinfluB auf die chemische Verwandtschaft der Metalloide untereinander wird dadurch ausgeübt, daB bei der ersten Horizontalreihe nur 2 Elektronen in dem Ringe des inneren Flektronensystems vorhanden sind ${ }^{1}$ ), während in den höheren Horizontalreihen eine gröBere Zahl Elektronen in jedem Elektronenringe des inneren Systems vorhanden ist. So stehen bei zwei miteinander verbundenen Atomen eines Elements der zweiten Horizontalreihe fünf negative Elektronen des einen Atoms fünf positiven Stellen des anderen Atoms gegenüber: während bei einem Element der ersten Horizontalreihe wie gesagt nur zwei negative Elektronen des einen Atoms zwei positiven Stellen des anderen Atoms im Molekül einander gegenüberstehen. Demnach würden die Atome bei den Elementen der ersten Horizontalreihe sich weniger fest miteinander verbinden, wenn nicht noch folgender Umstand hinzukäme: Die Kraftlinien, die von dem Atomkern des einen Atoms zu den Elektronen des anderen Atoms gehen, haben, wie der punktierte Pfeil in Fig. 7 zeigt, bei den Elementen der ersten Horizontalreihe einen direkten, kurzen und ungehinderten Weg. Bei den folgenden Horizontalreihen wird dagegen ein größerer Teil der vom positiven Atomkern ausgehenden Kraftlinien von den seitlichen inneren Elektronenringen des eigenen Systems aufgefangen (vgl. den punktierten Pfeil in Fig. 8).

1) A. a. O. S. $20 \overline{5}$.

2. anorg, a. Allg. Chem. Bd. 108. 
Dieser Unterschied in der Atomkupplung zwischen den Elementen der ersten Horizontalreihe einerseits und bei den Elementen der folgenden Horizontalreihen andererseits führt zu bemerkenswerten Wirkungen, wenn sich ein Metalloid der ersten Horizontalreihe mit einem Metalloid einer der folgenden Horizontalreihen verbindet, z. B. Sauerstoff mit Schwefel. In diesem Falle können zwar nicht die vom S-Atom zum O-Atom gehenden Kraftlinien, wohl aber die vom 0-Atom zum S-Atom gehenden Kraftlinien den durch den Pfeil in Fig. 7 angedeuteten kurzen Kraftlinienweg ungehindert benutzen. Da die inneren Elektronenringe in der zweiten Horizontalreihe fünf Elektronen haben, stehen dem positiven Sauerstoffatomkern in seinen Schwefelverbindungen fünf negative Elektronen gegenüber, die Anziehungskräfte auf ihn ausüben, während ihm bei einem Elemente der ersten Horizontalreihe nur zwei Elektronen des anderen Atoms gegenüberstehen. Demuach wird ein Sauerstoffatomkern oder ein Atomkern eines anderen Metalloids der ersten Horizontalreihe von einem gegenüberstehenden Atom der zweiten Horizontalreihe stärker angezogen als von einem anderen gegenüberstehenden Atom der ersten Horizontalreihe. Aus diesem Grunde wird ein O- oder Fl-Atom von einem $\mathrm{Si}$-Atom stärker angezogen als von einem C-Atom, von einem P-Atom stärker als von einem N-Atom, von einem S-Atom stärker als von einem O-Atom und ron einem ClAtom stärker als von einem Fl-Atom.

Ferner wird die chemische Verwandtschaft noch dadurch beeinfluBt, daB sich die Elektronen bei den verschiedenen Elementen mit verschiedener Geschwindigkeit um ihren Atomkern drehen. Verbinden sich die Atome verschiedener Elemente, so bremsen die langsamer kreisenden Elektronen des einen Elements die schneller kreisenden Elektronen des anderen Elements und umgekehrt. Bei ungleich bleibender Winkelgeschwindigkeit würde auch das Molekül nur wenig beständig sein können, weil sich die in Fig. 3 angedeutete Lage der negativen Elektronen des einen Atoms zu den positiven Stellen des anderen Atoms fortwährend ändern würde, wodurch eine Störung der das Atom zusammenhaltenden Kräfte eintreten würde.

Vorhin wurde abgeleitet, daB ein O-Atom und ein Fl-Atom sich wegen der großen Zahl, der in ihren äußeren Elektronenringen enthaltenen Elektronen nicht miteinander verbinden können. Dagegen können sich zwei Sauerstoffatome und zwei Fluoratome miteinander zu Sauerstoffimolekülen bzw. Fluormolekülen verbinden. Dies kommt daher, daB die Winkelgeschwindigkeit der Elektronen bei den beiden 
sich verbindenden $\mathrm{O}$-Atomen bzw. bei den beiden sich verbindenden Fl-Atomen gleich groß ist, so daß für die Ausgleichung der verschieden großen Elektronengeschwindigkeiten keine Kräfte mehr erforderlich sind.

\section{Verbindungen zwischen Metallatomen und Metalloidatomen.}

Wenn sich ein Metallatom und ein Metalloidatom zu einem Molekül verbinden, so vereinigt sich die Kupplung für Metalloidatome nach Fig. 3 mit der Kupplung für Metallatome nach Fig. 4 zu einer neuen Atomkupplung, die in Fig. 9 veranschaulicht ist.

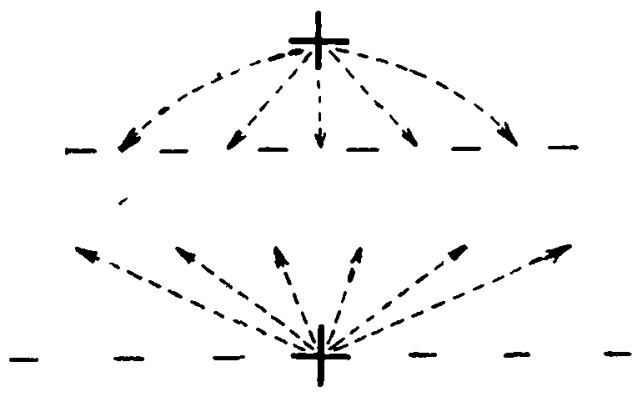

Fig. 9.

Aus einem Metallatom und einem Metalloidatom bestehendes Molekül.

Das Wesen dieser Kupplung kommt dadurch zustande, daB die Elektronen des Metallatoms stärker zurückgedrängt werden, als die Elektronen des Metalloidatoms.

Bei dieser Kupplung nach Fig. 9 wird das Metallatom von dem inneren Elektronensystem des Metalloidatoms um so stärker angezogen, je gröBer das am Metallatom entstandene positive Feld ist, d. h. je stärker die Elektronen des Metallatoms zurückgedrängt sind. Umgekehrt wird das Metalloidatom ron dem positiven Feld des Metallatoms um so stärker angezogen, je näher die Elektronen des Metalloidatoms ihm gegenüber stehen, $d . h$. je weniger das innere Elektronensystem des Metalloidatoms zurückgedrängt ist. Nun können aber die Elektronen des Metallatoms um so stärker verschoben werden, je mehr das betreffende Metall links steht, und die Elektronen des Metalloidatoms können um so weniger leicht verschoben werden, je mehr das betreffende Metalloid rechts steht. ${ }^{3}$ Daher muß die Verwandtschaft zwischen einem Metall und einem

1) a. a. O. S. $196-197$. 
Metalloid um so größer sein, je mehr links das erstere und je mehr rechts das zweite im periodischen System steht.

Diese Regel kommt in den ersten Horizontalreihen des periodischen Systems deutlich zum Ausdruck. In den folgenden Horizontalreihen werden ihre Wirkungen durch andere Einflüsse mitunter abgeändert.

In der dritten und fünften Horizontalreihe treten überbaupt keine Metalloide auf, weil in diesen beiden Horizontalreihen die Elektronen der äußeren Elektronenringe aus den von mir früher abgeleiteten Gründen leicht verschiebbar sind und infolge ihrer Verschiebbarkeit metallische Eigenschaften in ihren Elementen hervorrufen. ${ }^{1}$ )

Die Elemente der vierten und sechsten Horizontalreihe erhalten ihre Eigenart dadurch, daB am Äquator der von ihrem inneren Elektronensystem gebildeten Kugel kein Elektronenring vorhanden ist. ${ }^{2}$ )

Verbindet sich nun ein Metall aus der vierten oder sechsten Horizontalreihe mit einem S- oder O-Atom, so werden die dem O-Atom gegenüber liegenden Elektronen des Metallatoms nach dem Äquator hingedrängt, wodurch sich ihre Zentrifugalkraft erhöht. Die zu dieser Erhöhung der Zentrifugalkraft erforderliche Arbeit muB von den Anziehungskräften geleistet werden, die dás Metallatom und das O-Atom verbinden. Diese Anziehungskräfte werden dementsprechend vermindert. Bei den Metallen der dritten, fünften, siebenten Horizontalreihe werden zwar auch ein oder mehrere Elek-

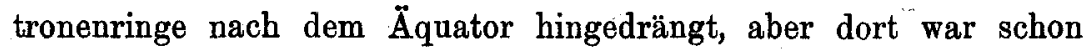
vorher ein Elektronenring auf dem $\ddot{A} q u a t o r$ vorhanden, der von demselben abgedrängt wird, wobei sich die Zentrifugalkraft seiner Elektronen vermindert. Die Durchschnittszentrifugalkraft des inneren Elektronensystems wird daher bei den Elementen der dritten, fünften, siebenten Horizontalreihe weniger erhöht als bei den Elementen der vierten und sechsten Horizontalreihe. Deshalb wird bei den ersteren ein geringerer Teil der Anziehungskräfte eines O-Atoms für die Erhöhung der Zentrifugalgeschwindigkeit verbraucht. Aus diesem Grunde kommen die Anziehungskräfte, die ein O-Atom oder ein S-Atom auf Metallatome ausübt, in der dritten, fünften, siebenten Horizontalreihe, also beim $\mathrm{Ca}, \mathrm{Sr}, \mathrm{Ba}$ stärker zur Geltung als in der vierten, sechsten Horizontalreihe, z. B. beim Zn und Cd.

1) a. a. O. S. 198 u. 200.

2) a. a. O. S. 199 u. 200 . 
Wir wollen jetzt die Sauerstoffverbindungen und Schwefelverbindungen der Leicht- und Schwermetalle miteinander vergleichen. Dabei müssen wir uns wieder daran erinnern, daB beim Sauerstoffatom nur ein innerer Elektronenring vorhanden ist, der mit dem positiven Atomkern in einer Ebene liegt, während beim Schwefelatom dagegen zu beiden Seiten der durch den Atomkern gehenden Mittelebene des Atoms je ein Elektronenring des inneren Systems liegt (vgl. Figg. 5-8). Infolge dieses Unterschieds werden die vom Atomkern ausgehenden Kraftlinien beim Sauerstoff weniger als beim Schwefel gehindert, zu den Valenzelektronen und zu den Elektronen eines gegenüberstehenden Atoms zu gelangen (vgl. Fig. 10 mit

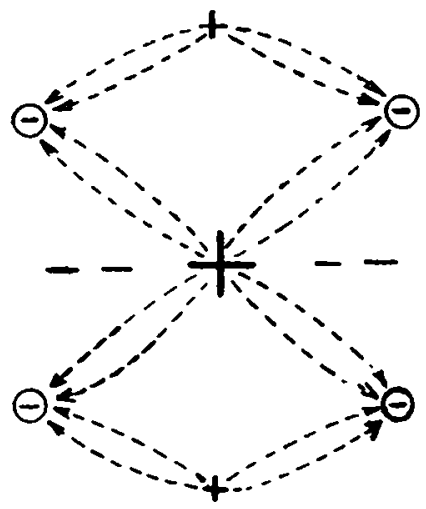

Fig. 10. $\mathrm{H}_{2} \mathrm{O}$-Molekül.

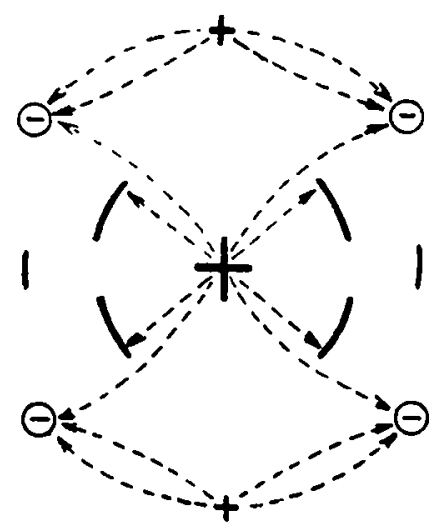

Fig. 11. $\mathrm{H}_{8} \mathrm{~S}-$ Molekỉll.

Fig. 11). Diese werden daher vom Sauerstoffatomkern stärker angezogen als vom Schwefelatomkern und in Übereinstimmung hiermit haben die Sauerstoffverbindungen der leichteren Metalle und des Wasserstoffs eine gröBere Verbindungsenergie als deren Schwefelverbindungen. Dagegen sind bei den Schwermetallen die Schwefelverbindungen beständiger als die Sauerstoffverbindungen, was in folgender Weise zu erklären ist. Die Atome der Schwermetalle haben eine gröbere Anzahl Elektronenringe, die verhindern, daB vom Atomkern des Schwermetalls genügend Kraftlinien zu den Valenzelektronen gelangen, um diese kräftig festzuhalten. Diese inneren Elektronenringe des Schwermetallatoms werden bei den Schwefelverbindungen stärker zurückgedrängt als bei den Sauerstoffverbindungen, da die 10 inneren Elektronen des Schwefelatoms eine erheblich stärkere AbstoBungskraft ausüben als die zwei inneren Elektronen eines Sauerstoffatoms. Infolgedessen können bei den Schwefel- 
verbindungen der Schwermetalle mehr Kraftlinien zu den Valenzelektronen gelangen als bei den entsprechenden Sauerstoffverbindungen (vgl. das $\mathrm{HgO}-$ Molekül in Fig. 12 mit dem HgS-Molekül in Fig. 13).

Bei den Chlorverbindungen können die Elektronenringe eines zweiwertigen Schwermetalls nicht in dem MaBe wie bei den Schwefelverbindungen zurückgedrängt werden, da sich auf jeder der beiden Seiten des Metallatoms ein Chloratom befindet. Dagegen sind die Chlorverbindungen aus einem anderen Grunde beständiger als die Sauerstoffverbindungen. Da Sauerstoff zweiwertig auftritt, kreisen bei den Sauerstoffverbindungen vier Valenzelektronen im Valenzelektronenring, von denen je zwei von dem betreffenden Metallatom

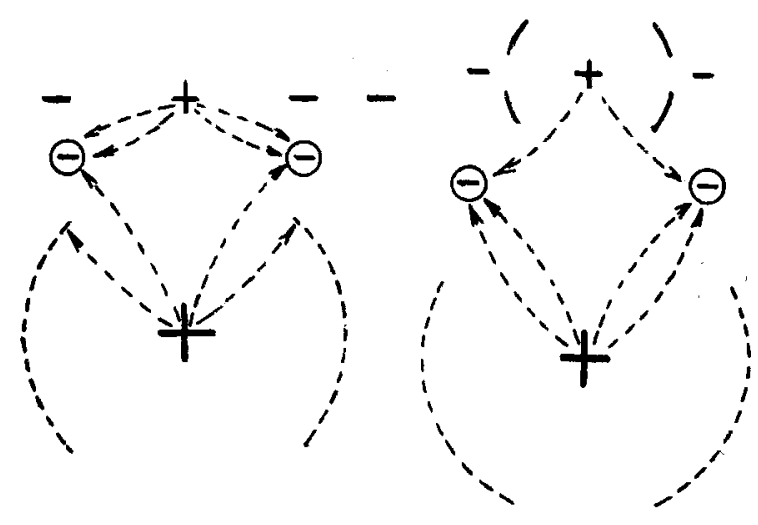

Fig. 12. $\mathrm{HgO}$.

Fig. 13. HgS.

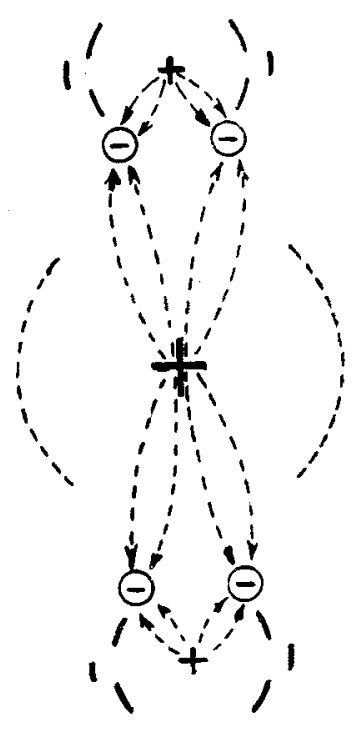

Fig. 14. $\mathrm{HgCl}^{*}$.

stammen. Da das Chlor dagegen einwertig ist, kreisen bei ihm in jedem Valenzelektronenringe nur zwei Valenzelektronen, von denen das eine vom Chloratom, das andere von dem betreffenden Metallatom stammt. Infolge dieser geringeren Zahl von Valenzelektronen üben die Valenzelektronenringe bei den Chlorverbindungen weniger AbstoBungskräfte aus (vgl. S. 141) und haben auBerdem einen geringeren Durchmesser als bei den Sauerstoffverbindungen. Dieser kleinere Durchmesser der Valenzelektronenringe hat zur Folge, daß von den Kraftlinien, die vom Atomkern eines Schwermetalls ausgehen, bei den Chlorverbindungen ein größerer Teil zu den Valenzelektronen gelangt als bei den Sauerstoffverbindungen, vgl. Fig. 14. Daher sind die Chloride der schweren Metalle beständiger als deren Oxyde. 


\section{Die Entstehang der Ionen.}

Wir wollen davon ausgehen, daB die Spaltung eines Salzes in Ionen durch StöBe verursacht wird, die die zerfallenden Salzmoleküle durch andere in schneller Bewegung befindliche Moleküle erhalten. Infolge dieser Stöße erfolgt die Spaltung der Metallsalze in Ionen durch seitliche Verschiebung des Anions zum Kation. Wir müssen daher zunächst untersuchen, unter welchen Umständen zwei miteinander verbundene Atome durch seitliche Verschiebung voneinander getrennt werden können.

Bei der in Fig. 3 veranschaulichten Kupplung zweier Metalloidatome wird eine 'Trennung der miteinander verbundenen Atome durch seitliche Verschiebung dadurch verhindert, daB abwechselnd positive und negative Stellen einander gegenüberstehen. Diese Wirkung der aufeinander abwechselnd folgenden positiven und negativen Stellen wird bei einer Verbindung zweier Metallatome durch den bei Fig. 4 abgeleiteten positiven Zwischenraum fast ganz aufgehoben, wodurch der bereits im Kapitel 2 erwähnte leichte Zerfall der Metallmoleküle in ihre Atome wesentlich erleichtert wird. Bei der Verbindung eines Metallatoms mit einem Metalloidatom wird dieser positive $Z$ wischenraum weniger grob, weil nur die Elektronen des Metallatoms, nicht aber die des Metalloidatoms zurückgedrängt werden. Deshalb wird der Widerstand gegen seitliche Verschiebung, der durch den Wechsel der einander gegenüberstehenden positiven und negativen Stellen entsteht, bei der Bindung eines Metalls mit einem O- oder S-Atom noch nicht ganz aufgehoben und genügt noch, um eine Trennung der Atome zu verhindern. Wenn sich aber ein Metallatom mit einem Halogenatom verbindet, so entstehen andere Verhältnisse dadurch, daB bei den Halogenen ein ÜberschuB der positiven Ladungen am Atomkern über die negativen Elektronen des inneren Systems vorhanden ist, der groB genug ist, um die Valenzelektronen ganz in die Sphäre des Halogenatoms zu ziehen.

Dieser Überschub der positiven Ladungen am Atomkern kommt in folgender Weise zustande. Da ein Atom als ganzes neutral ist, muß die Gesamtzahl der positiven Ladungen gleich sein der Gesamtzahl der zum Atom gehörenden Elektronen. Da aber ein Teil dieser Elektronen aus dem inneren Elektronensystem heraus zu den Valenzstellungen oder in den äuBeren Elektronenring geht, so bleibt beim Atomkern ein ÜberschuB von positiven Ladungen, der beim C-Atom 4, beim N-Atom 5, beim O-Atom 6 und bei einem Halogenatom 7 positive Ladungen beträgt. Ist nun ein Halogenaton mit einem 
Metallatom verbunden, so wirkt der Überschuß von sieben positiven Ladungen an dem Atomkern des Halogenatoms so stark auf die zwischen dem letzteren und dem Metallatom kreisenden Valenzelektronen, daB diese ganz in die Sphäre des Halogenatoms hineingezogen werden.

DaB dieses Hineinziehen der Valenzelektronen nur dann erfolgt, wenn das Halogenatom einem Metallatom gegenübersteht, nicht aber, wenn es einem Metalloidatom gegenübersteht, geschieht aus folgenden Gründen:

Bei den Metalloidatomen ist der Atomkern von den Elektronen seines inneren Elektronensystems umgeben, so da $B$ nur relativ wenige der von dem Atomkern ausgehenden Kraftlinien zu den Valenzelektronen gelangen. Sind daher in einem Molekül nur Metalloid. atome vorhanden, so werden die Valenzelektronen mit relativ ge-

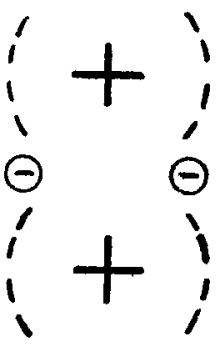

Fig. 15.

Stellung der Valenzelektronen zwischen zwei Metalloidatomen.
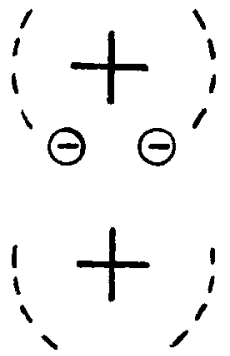

Fig. 16. Stellung der Valenzelektronen $z$ wischen einem Metall- und einem Halogenatom.

ringen Kräften angezogen und kreisen daher in relativ großer Entfernung um die Mittelachse ihres Moleküls (vgl. Fig. 15). Ist dagegen auch ein Metallatom im Molekül, so sind die inneren Elektronen desselben (wie bei Fig. 4 und 9 gezeigt) zurückgedrängt. Daher gelangt ein gröBerer Teil der von dem Metallatomkern ausgehenden positiven Kraftlinien zu den Valenzelektronen und zieht diese näher an die Mittelachse des Moleküls heran. Nachdem sie aber erst näher an diese Mittellinie herangezogen sind, werden sie, falls das Metallatom mit einem Halogenatom verbunden ist, von dem dort am Atomkern vorhandenen ÜberschuB positiver Ladungen stärker angezogen als beim Metallatom und gelangen so in die Sphäre des Halogenatoms etwa in die aus Fig. 16 ersichtliche Stellung. In dieser Stellung sind sie so weit von dem Metallatom fortgerückt, daB ihre negativen Kraftlinien die positiven Stellen am Rande des Halogenatoms völlig überdecken, und daB die ganze Seite des Halogenatoms negativ wird. Diese negative Seite des Halogenatoms 
zieht dann die positive Seite des Metallatoms an, so daB diese beiden Atome so nahe zusammengezogen werden, bis die AbstoBungskräfte, die die inneren Elektronensysteme gegeneinander ausüben, eine weitere Annäherung der beiden Atome verhindern.

Die Valenzelektronen treten daher za den Halogenatomen nicht erst bei dem Zerfall des Moleküls in Ionen, sondern schon bei der Bildung. des Halogensalzes. Diese aus der hier entwickelten Theorie abgeleitete Folgerung wird dadurch bestätigt, daß DEBYE und SCHERRER mit Hilfe von Röntgenwellen feststellen konnten, da $B$ in einem Lithiumfluoridkristall das Li-Atom ein Elektron weniger und das Fl-Atom ein Elektron mebr hat als im neutralen Zustande. $\left.{ }^{1}\right)$ Infolge dieser Verschiebung der Valenzelektronen vom Metallatom zum Säureradikal fehlen bei dem letzteren die einander gegenüber stehenden abwechselnd positiven und negativen Stellen, die eine seitliche Verschiebung der Atome zueinander verhindern. Daher können jetzt das Halogenatom und das Metallatom durch seitliche Verschiebung, die durch den StoB anderer in schneller Bewegung befindlicher Moleküle verursacht werden kann, voneinander getrennt werden. Bei dieser Trennung bleiben dann die Valenzelektronen in der in Fig. 16 gezeichneten Stellung beim Halogenatom und machen dieses zum negativen Kation.

Die Atome der Halogene können nach dem Vorhergehenden also deshalb Säureradikale bilden, weil bei ihnen der Überschuß der am Atomkern vorhandenen positiven Ladungen über die negativen Ladungen so groB ist, daB er die Valenzelektronen in der eben beschriebenen. Weise von dem Metallatom fort und an das Halogenatom heran reißen kann. Bei den anderen Metalloiden ist dieser ÜberschuB der positiven Ladungen über die negativen Ladungen des inneren Elektronensystems weniger groB, so da $B$ der ÜberschuB der positiven Ladungen eines einzigen Atoms nicht genügt, um die Valenzelektronen von einem mit ihm verbundenen Metallatom fortzureißen. Aber es können sich mehrere Metalloidatome in der Weise miteinander verbinden, daB die Überschüsse der in ihnen enthaltenen positiven Ladungen sich gegenseitig so verstärken, daß die Kombination dieser Überschüsse ausreicht, um die Valenzelektronen vom Metallatom fort an sich heranzuziehen. In diesem Falle entsteht ein Säureradikal. Z. B. hat ein C-Atom einen ÜberschuB von vier positiven Einheiten im Atomkern, ein N-Atom einen Überschuß von fünf positiven Einheiten im Atomkern; bei der

1) Phys. Zeitschr. 19 (1918), 474. 
Verbindung dieser beiden Atome kombinieren sich die Wirkungen dieser beiden Überschüsse positiver Ladungen zu einer Gesamtwirkung, die hinreicht, um die schwach sauren Eigenschaften des $\mathrm{CN}$.Radikals zu erzeugen. Bei den Radikalen $\mathrm{NO}_{3}, \mathrm{SO}_{4}$ usw. wird die Gesamtwirkung stärker, weil in diesen Radikalen mehr Atome mit größeren Überschüssen positiver Ladungen enthalten sind.

Dagegen hat das $\mathrm{NH}_{4}$-Radikal die Eigenschaften einer Base, weil das 5. Valenzelektron des $\mathrm{N}$-Atoms nur noch lose von diesem festgehalten wird und daher durch den bei einem Säureradikal vorhandenen ÜberschuB positiver Ladungen rom $\mathrm{NH}_{4}$-Radikal an sich gerissen werden kann.

\section{Wasserstoffverbindungen.}

Der Wasserstoff erhält seine Eigenschaften dadurch, daB er nur Valenzelektronen aber kein inneres Elektronensystem besitzt. Infolge Fehlens des letzteren liegt sein positiver Atomkern den Elektronen anderer Atome, die mit ihm zu einem Molekül verbunden sind, direkt gegenüber und spielt daher eine gleiche Rolle, wie das positive Feld, das bei den Metallatomen durch Zurückdrängung der inneren Elektronen entsteht, wie hier oben im Kapitel 2 und 4 näher ausgeführt wurde. Aus diesem Grunde verhält sich ein Wasserstoffatom in vielen chemischen Verbindungen wie ein Metallatom. Unterschiede entstehen aber dadurch, daß das positive Feld beim Wasserstoffatom von vornherein vorhanden ist, während es bei den Metallatomen erst durch Zurückdrängung der zum Metallkern gehörenden Elektronen geschaffen werden muß.

Die Fähigkeit, die Elektronen eines gegenüberliegenden Atoms zurückzudrängen, wächst nun bei den Metalloiden innerhalb jeder Horizontalreihe von links nach rechts (vgl. S. 147).

Unter den Metalloiden der ersten Horizontalreihe ist daher die Fähigkeit, die Elektronen eines Metallatoms zurückzudrängen, beim Kohlenstoff und Stickstoff am schwächsten entwickelt. Diese beiden Metalloide gehen daher mit den Metallen viel weniger leicht Verbindungen ein als mit dem Wasserstoff, weil eben im H-Atom keine inneren Elektronen vorhanden sind, die erst zurückgedrängt werden müssen, damit das positive Feld des H-Atomkerns den negativen Elektronen des C- bzw. N-Atoms direkt gegenüber liegt und von diesen angezogen und festgehalten wird.

In den Sauerstoffverbindungen werden die dem O.Atom gegenüberstehenden Elektronen der Metallatome bei den Alkalimetallen 
und den Metallen der alkalischen Erden soweit zurückgedrängt, daB ein positives Feld entsteht, welches stärker ist, als das positive Feld des H-Atomkerns, der ja nur eine positive Ladung hat. Daher hat das O-Atom zu den Alkalimetallen und den Metallen der alkalischen Erden eine größere Verwandtschaft als zum Wasserstoff. Bei den übrigen Metallen sind die Elektronen weniger leicht zurückzudrängen, so dab das bei diesen entstehende positive Feld schwächer bleibt als das des H-Atomkerns. Bei diesen Metallen ist daher die Verwandtschaft zum Sauerstoff geringer als die Verwandtschaft des Wasserstoffs zum Sauerstoff.

Bei den Halogenen ist die Fähigkeit, die Elektronen der Metallatome zurückzudrängen, am stärksten. Deshalb werden bei einer größeren Anzahl von Metallen die Elektronen des Metallatoms von einem gegenüberstehenden Chloratom so weit zurückgedrängt, daß das dadurch entstehende positive Feld stärker wird, als das der einen positiven Ladung des H-Atomkerns. Deshalb hat das Chlor zu vielen Metallen eine gröBere Verwandtschaft als zum Wasserstoff. Auf diese Weise erklärt es sich, weshalb bestimmte Metalle, wie z. B. das Zink, den Wasserstoff zwar aus einem HCl-Molekül, nicht aber aus einem $\mathrm{H}_{2} \mathrm{O}$-Molekül austreiben können.

Die 10 Elektronen des inneren Elektronensystems am S-Atom haben genügend Kraft, um die Elektronen bei allen Metallatomen zurückzudrängen; die zwei inneren Elektronen des Sauerstoffatoms haben noch nicht soviel Kraft. Daher kann der Wasserstoff aus dem $\mathrm{H}_{2} \mathrm{~S}$-Molekül von allen Metallen verdrängt werden, aus dem $\mathrm{H}_{2} \mathrm{O}$ Molekül aber nur von solchen Metallen, bei denen die Elektronen besonders leicht zurückgedrängt werden können.

\section{Beziehungen zwischen den Valenzelektronen und den Gerüchen der chemischen Verbindungen.}

Es muB nun noch untersucht werden, welchen Einflub die abwechselnd positiven und negativen Stellen, die nach Fig. 3 bei zwei Metalloidatomen einander gegenüberstehen, auf die zwischen den beiden Atomen kreisenden Valenzelektronen ausüben. Die ursprüngliche Bahn dieser Valenzelektronen sei in Fig. 17 durch eine gestrichelt gezeichnete Linie angedeutet. Wenn sich nun eines. der Valenzelektronen z. B. gerade zwischen einer positiven Stelle des oberen Atoms und einem negativen Elektron des unteren Atoms befindet, so wird es von der ersteren angezogen und von dem letzteren abgestoBen. Es wird daher aus 
seiner (gestrichelt gezeichneten) ursprünglichen Bewegungsbahn abgelenkt in eine Richtung, welche durch die Komponente seiner Zentrifugalkraft und der eben angegebenen Anziehungs- und AbstoBungskräfte der beiden Atome bestimmt wird. Wenn es in Richtung dieser Komponente dauernd weiter fliegen könnte, würde es in das Innere des oberen Atoms hineinfliegen, weil es sich zufällig gerade ciner positiven Stelle desselben gegenüber befunden hatte. Aber bevor es dieses Atom erreicht, haben sich die beiden Atome weiter gedreht, wodurch das Elektron zwischen einem negativen Elektron des oberen und einer positiven Stelle des unteren Atoms gerät und nun anstatt nach oben nach unten hin abgelenkt wird. Auf diese Weise wird jedes einzelne Valenzelektron abwechselnd nach oben und nach unten hin ab-

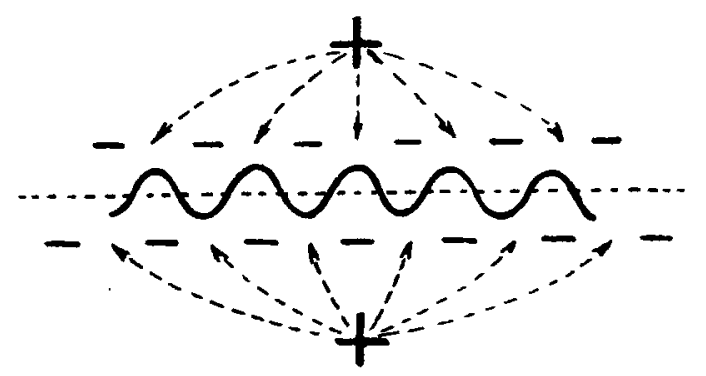

Fig. 17.

Schwingungen der Valenzelektronen zwisehen zwei Metalloidatomen.

gelenkt und es entstehen die aus Fig. 17 ersichtlichen Schwingungen der Valenzelektronen.

Die folgenden Ausführungen lassen die Annahme berechtigt erscheinen, daB die Gerüche der chemischen Verbindungen durch die eben abgeleiteten Schwingungen der Valenzelektronen hervorgerufen werden.

Läge die bisher unbekannte Ursache der Geruchsentstehung im Innern der Atome, so müBte ein und dasselbe Element in verschiedenen Verbindungen einen gleichartigen Geruch hervorrufen und diesen Geruch auch als Element aufweisen. Da dies nicht der Fall ist, bleibt nichts anderes übrig als die Annahme, daB der Geruch einer chemischen Verbindung durch das Zusammenwirken mehrerer Atome im Molekül erzeugt wird, und zwar in der Weise, daB jedes der Atome EinfluB auf die Eigenart des erzeugten Geruchs hat. Dieser Bedingung entsprechen die eben abgeleiteten Elektronenschwingungen, denn sie werden innerhalb des Moleküls von den Atomen in der Weise erzeugt, daB jedes der Atome Ein- 
fluB auf die Eigenart der erzeugten Schwingungen hat. Ferner habe ich bereits in einigen früheren Arbeiten ${ }^{1}$ ) gezeigt, daB man auch die Entstehung der Geruchsempfindung in den Riechnerven am besten durch die Annahme erklären kann, daß in den Molekülen der riechenden Körper elektrische Schwingungen vorhanden sind, die andere in den Riechnerven vorhandene Schwingungen durch Induktionswirkung und Resonanz verstärken, wenn riechende Körperteile mit der Atemluft in die Nase gezogen werden.

Demnach ist also der Geruch eine physikalische Erscheinung, die, ähnlich wie das Licht, auf elektrischen Schwingungen, nämlich auf den hier abgeleiteten Schwingungen der Valenzelektronen, beruht.

Diese Erklärung des Geruches gibt uns ein Mittel in die Hand, mit dessen Hilfe die im Vorhergehenden abgeleiteten Folgerungen über den inneren Aufbau der Atome und Moleküle nachgeprüft werden können. Denn, wenn der Geruch durch die hier abgeleiteten Elektronenschwingungen der Valenzelektronen verursacht wird, so müssen alle diejenigen chemischen Verbindungen geruchlos sein, in deren Molekülen keine negativen und positiven Stellen, die die Valenzelektronen in Schwingungen versetzen können, miteinander abwechseln.

Nach den Ausführungen des Kapitel 2 können diese positiven und negativen Stellen nur bei Metalloiden, nicht aber bei Metallatomen mit genügender Kraft auf die Valenzelektronen einwirken und diese in stärkere Schwingungen versetzen. Demnach haben die Metalle auch nur kaum wahrnehmbare oder gar keine Gerüche, und die deutlich wahrnehmbaren Gerüche treten lediglich bei denjenigen Verbindungen auf, in deren Molékülen mehrere Metalloidatome miteinander verbunden sind. Dabei ist es auch noch von Bedeutung, ob die Metalloidatome durch eine oder mehr Valenzen miteinander verbunden sind. Denn bei mehrwertigen Bindungen ist die Zahl der zwischen den Atomen kreisenden Valenzelektronen gröBer als bei einwertigen, und es erfordert einen gröBeren Aufwand von Energie, 4 Valenzelektronen in Schwingungen zu versetzen als nur 2 Valenzelektronen. Außerdem werden noch die zu den Atomen gehörenden Elektronen bei mehrwertigen Verbindungen stärker zurückgedrängt (vgl. hier oben S. 147 u. 154), so daB auch die Kräfte, die 'die Valenzelektronen in Geruchsschwingungen versetzen können, bei zweiwertigen

1) Biologisches Zentralblatt 1913, S. 716 u. f.; Prometheus Nr. 1282 (Jahrg. XXV, Nr. 34, S. 529); Wochenschrift für Brauerei 1918, Nr. 15-17; Prometheus Nr. 1535 u. 1536; Jahrg. XXX, Nr. 26, S. 201 u. Nr. 27, S. 209. 
Verbindungen kleiner sind als bei einwertigen. Dementsprechend sind die Bedingungen für die Entstehung von Gerüchen am günstigsten, wenn einwertige Bindungen zwischen den Matalloidatomen vorhanden sind. In Übereinstimmung biermit haben bei den Elementen überhaupt nur die einwertigen Metalloide einen deutlich wahrnehmbaren Geruch.

Bei den Oxyden und Sulfiden der Metalle ist, wie F'ig. 9 erkennen läBt, zwar nur der Teil des Raumes zwischen den Atomen, der sich in der Nähe des Metallatoms befindet, gleichmäBig elektrisch positiv, aber die Valenzelektronen werden in diesen elektrisch positiven Teil hineingezogen und kreisen in diesem, so daB der Wechsel der positiven und negativen Stellen im O- bzw. S-Atom sie nicht in Schwingungen versetzen kann. Daher sind die Metalloxyde und Sulfide geruchlos.

Phosphor und Selen zeigen als Elemente metallische Modifikationen und müssen also zurückdrängbare Elektronen haben, diese metallische Eigenschaft.ihrer Atome ist offenbar die Ursache dafür, daß ihre Oxyde im Gegensatz zu den Oxyden des Stickstoffis und Schwefels geruchlos sind, wie die Oxyde der anderen Metalle. Dagegen haben die Wasserstoffverbindungen des Phosphors und Selens einen Geruch, weil das H-Atom kein positives Feld hat, das groB genug wäre, um die Bahn der Valenzelektronen zu umfassen.

Das Kohlenstoffatom ist weniger als ein Sauerstoff- oder Schwefelatom geeignet, um die Elektronen der Metalle zurückzudrängen (vgl. hier oben S. 147 u. 154). Infolgedessen entsteht in den Fällen, in denen ein Metallatom mit einem Kohlenstoffatom verbunden ist, kein positives Feld am Metallatom, das grob genug wäre, um die Valenzelektronen in sich aufzunehmen und so zu verhindern, daB sie in Schwingungen versetzt werden, deshalb haben viele metallorganische Verbindungen einen Geruch.

Verbindet sich ein Metallatom und ein Säureradikal miteinander, so werden nach den Ausführungen des Kapitels 5 die Valenzelektronen an das Säureradikal herangerissen; dadurch erhält dieses einen ÜberschuB negativer Elektrizität, der die positiven Stellen an den Rändern der verschiedenen Atome, die das Säureradikal zusammensetzen; soweit ausgleicht, daB kein Wechsel ron positiven und negativen Stellen mehr übrig bleibt, der die Valenzelektronen in Schwingungen versetzen könnte. Aus diesem Grunde wird jedes Säureradikal geruchlos, sobald es sich mit einem Metallatom verbindet oder als Ion auftritt. 
Auch das $\mathrm{NH}_{4}$-Radikal ist geruchlos, weil ihm eines der zum $\mathrm{N}$-Atom gehörenden Valenzelektron entrissen wurde, so daB in diesem Atomkomplex ein Überschuß positiver Elektrizität zurückgeblieben ist, der die negativen Stellen am Rande des N-Atoms soweit ausgleicht, daB die zwischen ihm und den mit ihm verbundenen H-Atomen kreisenden Valenzelektronen nicht mehr in Schwingungen gesetzt werden.

Bei den Verbindungen des Wasserstoffs mit Metalloiden kommen die Valenzelektronen bei ihrem Kreislauf abwechselnd den positiven und negativen Stellen des Metalloidatoms gegenüber zu stehen und werden daher in Schwingungen gesetzt. In Übereinstimmung hiermit haben die Verbindungen des Wasserstoffs mit den Metalloiden in der Regel einen Geruch. Ausnahme von dieser Regel bilden:

1. Ganz regelmäBig gebaute Moleküle, wie $\mathrm{CH}_{4}$, weil dort gleichartige und entgegengesetzt gerichtete Schwingungen entstehen, deren Wirkungen sich gegenseitig aufheben.

2. Kompliziertere Moleküle, in denen eine große Anzahl verschiedener Schwingungen entsteht, die miteinander interferieren und Perioden bilden, die zu kompliziert werden, um durch unsere Geruchsnerven noch wahrgenommen werden zu können. So ist z. B. bei den niederen Gliedern vieler homologer Reihen organischer Verbindungen ein deutlich wahrnehmbarer Geruch vorhanden, der bei den höheren Gliedern dieser Reihen verschwindet. Ebenso verschwindet der Geruch der Aldehyd- und Alkoholmoleküle, wenn diese sich zu Zuckermolekülen verbinden.

Im Gegensatz zu den Metallsalzen haben die Wasserstoffsäuren einen Geruch. Dieser Unterschied erklärt sich in folgender Weise. Bei der im Kapitel 5 abgeleiteten Wanderung der Valenzelektronen zum Säureradikal kann das Metallatom nicht mit in die Nähe des Säureradikals gezogen werden, weil sein inneres Elektronensystem dies verhindert. Der H-Atomkern aber kann infolge Fehlens eines inneren Elektronensystems zusammen mit den Valenzelektronen ziemlich nahe an das Säureradikal herangezogen werden. Die positive Ladung des H.Atomkerns eliminiert dann den EinfluB der negativen Ladungen der Valenzelektronen auf das Säureradikal so lange, bis das Molekül in Ionen zerfällt, wobei dann die Valenzelektronen beim Säureradikal verbleiben und dasselbe geruchlos machen.

Wie die eben angeführten Fälle zeigen, stimmt das Fehlen oder 
Vorhandensein eines Geruchs bei der groBen Masse der chemischen Verbindungen und Elemente völlig mit der hier entwickelten Theorie überein. Diese İbereinstimmung bildet zweifellos einen Beweis dafür, dab die hier aus der Elektronentheorie abgeleitete Verteilung positiver und negativer Stellen an den Rändern der Atome wirklich vorhanden ist und sowohl die chemische Verwandtschaft als auch die Entstehung der Gerüche in der hier abgeleiteten Weise regelt.

\section{Charlottenburg $I V$.}

(Bei der Redaktion eingegangen den 14. April 1919.) 